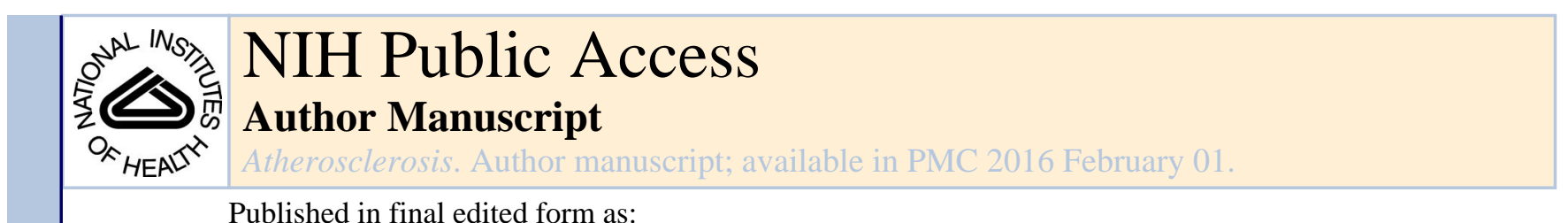

Published in final edited form as:

Atherosclerosis. 2015 February ; 238(2): 313-320. doi:10.1016/j.atherosclerosis.2014.12.024.

\title{
Insulin-like Growth Factor I Reduces Lipid Oxidation and Foam Cell Formation via Downregulation of 12/15-lipoxygenase
}

\author{
Sergiy Sukhanov ${ }^{*}, 1$, Patricia Snarski ${ }^{1}$, Charlotte Vaughn ${ }^{1}$, Patricia Lobelle-Rich ${ }^{1}$, Catherine \\ $\mathrm{Kim}^{1}$, Yusuke Higashi ${ }^{1}$, Shaw-Yung Shai ${ }^{1}$, and Patrice Delafontaine ${ }^{1}$ \\ ${ }^{1}$ Heart and Vascular Institute, School of Medicine, Tulane University, New Orleans, LA 70112
}

Abstract

Objective-We have shown that insulin-like growth factor I (IGF-1) infusion in Apoe ${ }^{-/-}$mice decreased atherosclerotic plaque size and plaque macrophage and lipid content suggesting that IGF-1 suppressed formation of macrophage-derived foam cells. Since 12/15-lipoxygenase (12/15LOX) plays an important role in OxLDL and foam cell formation, we hypothesized that IGF-1 downregulates 12/15-LOX, thereby suppressing lipid oxidation and foam cell formation.

\begin{abstract}
Approach and Results-We found that IGF-1 decreased 12/15-LOX plaque immunopositivity and serum OxLDL levels in Apoe ${ }^{-/-}$mice. IGF-1 reduced 12/15-LOX protein and mRNA levels in cultured THP-1 macrophages and IGF-1 also decreased expression of STAT6 transcription factor. IGF-1 reduction in macrophage 12/15-LOX was mediated in part via a PI3 kinase- and STAT6-dependent transcriptional mechanism. IGF-1 suppressed THP-1 macrophage ability to oxidize lipids and form foam cells. IGF-1 downregulated 12/15-LOX in human blood-derived primary macrophages and IGF-1 decreased LDL oxidation induced by these cells. IGF-1 reduced LDL oxidation and formation of foam cells by wild type murine peritoneal macrophages, however these effects were completely blocked in 12/15-LOX-null macrophages suggesting that the ability of IGF-1 to reduce LDL oxidation and foam cells formation is dependent on its ability to downregulate 12/15-LOX.
\end{abstract}

Conclusions-Overall our data demonstrate that IGF-1 reduces lipid oxidation and foam cell formation via downregulation of $12 / 15$-LOX and this mechanism may play a major role in the anti-atherosclerotic effects of IGF-1.

\section{Keywords}

Atherosclerosis; LDL/Oxidation/antioxidants; Lipoxygenase; Macrophages / monocytes; Oxidized lipids; Foam cells

\footnotetext{
*To Whom Correspondence Should be addressed: Sergiy Sukhanov, PhD, Heart and Vascular Institute, School of Medicine, Tulane University, 1430 Tulane Ave., SL-48, New Orleans, LA 70112, Phone: 504-988-8813, Fax: 504-988-4237, ssukhano@ tulane.edu. Publisher's Disclaimer: This is a PDF file of an unedited manuscript that has been accepted for publication. As a service to our customers we are providing this early version of the manuscript. The manuscript will undergo copyediting, typesetting, and review of the resulting proof before it is published in its final citable form. Please note that during the production process errors may be discovered which could affect the content, and all legal disclaimers that apply to the journal pertain.

DISCLOSURES

None
} 


\section{INTRODUCTION}

The "oxidative" hypothesis of atherogenesis postulates that transformation of low density lipoprotein (LDL) which is normally present in the circulation into its oxidized proatherogenic form (oxidized LDL, OxLDL) is a major driving event in atherogenesis [1]. OxLDL promotes lipid uptake by macrophages and formation of foam cells, the hallmark of atherosclerotic plaque [2]. LDL oxidation occurs via insertion of molecular oxygen into polyunsaturated fatty acids and this reaction is mediated by lipoxygenase enzymes [3]. Human 15-lipoxygenase, as well as mouse 12-lipoxygenase are capable of directly oxidizing esterified fatty acids in LDL particles. 15- and 12-lipoxygenases have high homology, share similar dual substrate specificity and are commonly referred to as 12/15-lipoxygenase (12/15-LOX) [3]. Disruption of the 12/15-LOX gene in both Apoe- and LDL receptordeficient mice markedly reduced atherosclerotic burden [4] [5] [6] showing that 12/15-LOX plays a dominant role in atherogenesis. It has been shown that macrophages are the primary source of 12/15-LOX in mouse tissues and that absence of macrophage-specific 12/15-LOX decreased the ability of macrophages to form foam cells and protected Apoe ${ }^{-/-}$mice from atherosclerosis [7].

We have shown previously that OxLDL is present in human advanced atherosclerotic plaques and that it co-localizes with areas of reduced expression of insulin-like growth factor I (IGF-1) and IGF-1 receptor [8] [9]. IGF-1 is an endocrine and autocrine/paracrine growth factor that has major effects on development, cell growth and differentiation [10]. IGF-1 reduces atherosclerosis burden and improves features of atherosclerotic plaque stability in $\mathrm{Apoe}^{-/-}$mice [11] [12] [13]. IGF-1-induced atheroprotection was associated with reduced plaque macrophages and plaque lipid levels [11] suggesting that IGF-1 suppressed foam cell formation in vivo. However molecular mechanisms mediating this effect were not identified. We also reported that growth hormone-releasing peptide 2 increased IGF-1 levels and markedly downregulated aortic 12/15-LOX mRNA and protein expression in Apoe - $^{--}$ mice [14]. In the current study we tested the hypothesis that IGF-1 downregulates macrophage 12/15-LOX expression thereby inhibiting transformation of macrophages into foam cells.

\section{MATERIALS AND METHODS}

\section{Cells}

Human THP-1 mononuclear cells (ATCC) were cultured in RPMI-1640 medium supplemented with $2 \%$ heat-inactivated fetal bovine serum and $0.05 \mathrm{mM} 2$-mercaptoethanol. THP-1 cells were differentiated into macrophages by treatment with $100 \mathrm{ng} / \mathrm{ml}$ phorbol myristate acetate (PMA) (Sigma). Mouse peritoneal macrophages were isolated 4 days after injection of $4 \%$ thioglycollate broth. Red blood cells were removed from peritoneal isolates by treatment with RBC lysis buffer (BioLegend), cells were washed with media, counted and plated at $1.2 \times 10^{6} / \mathrm{ml}$ in RPMI-1640 with $2 \%$ serum overnight to allow adherence. The majority of cells (>85\%) adhered to the plastic were considered to be macrophages based on morphology analysis after staining with Diff-Quick protocol and immunopositivity for Mac-3, CD36 and CD16 markers. Peripheral blood mononuclear cells (PBMC) were isolated from whole blood obtained from healthy donors (The Blood Center, New Orleans, 
LA) using Vacutainer CPT cell preparation tubes (BD Biosciences). PBMC were differentiated into macrophages by treatment with macrophage colony-stimulating factor (50 $\mathrm{ng} / \mathrm{ml}$ for 16h; Sigma) in RPMI-1640 medium.

\section{Animal Experiments}

All animal experiments were performed according to protocols approved by the Tulane's Institutional Committee for Use and Care of Laboratory Animals. Mice were housed individually and maintained on a 12:12-h light-dark cycle. Apoe ${ }^{-/-}$mice (C57BL/6, 8 weeks of age, Jackson Lab) were infused with saline or with human recombinant IGF-1 (1.5 $\mathrm{mg} / \mathrm{kg} /$ day, Tersica) using osmotic mini-pumps (ALZET, Cupertino, CA). IGF-1 administration protocol was selected based on our previous reports [1,2]. Mice were fed a high-cholesterol pro-atherogenic diet (Western-type diet, $42 \%$ of calories from fat) from Harlan-Teklad (TD 88137) for 4 or for 12 weeks. Human and mouse serum IGF-1 levels were measured at the end of the experiment with ELISA kits (R\&D Labs).

\section{Immunohistochemistry}

Serial sections $(6 \mu \mathrm{m})$ were taken throughout the entire aortic valve area (AVA) as per Paigen et al [3]. To quantify 12/15-LOX, sections were stained with rabbit polyclonal 15LOX antibody (Santa Cruz Biotechnology, H-235, 1:100 dilution) followed by incubation with biotinylated secondary antibody and avidin-peroxidase complex (Vectastain Elite ABC kit, Vector Laboratories Inc). Sections were developed with DAB substrate kit, and counterstained with hematoxylin. Antibody specificity was verified by staining of serial sections with "normal" IgG (obtained from an unimmunized animal of the same species as the primary antibody).

\section{Lipid Oxidation Assay}

THP-1-derived macrophages, PBMC-derived macrophages or mouse peritoneal macrophages were plated at $1.2 \times 10^{6} / \mathrm{ml}$ and incubated with/without IGF-1 in F10 media (Life Technologies) supplemented with $150 \mathrm{ug} / \mathrm{ml}$ human LDL (Kalen Biomedicals). To control for lipid autooxidation, media with LDL was incubated in parallel without cells. After $48 \mathrm{~h}$ incubation $5 \mathrm{mM}$ butylated hydroxytoluene (BHT) was added to conditioned media to prevent future lipid oxidation and samples were cleaned by centrifugation $(10,000$ $\mathrm{rpm} / 10 \mathrm{~min}$ ). Lipid oxidation levels were quantified with Lipid Peroxidation Microplate Assay Kit from Oxford Biomedical Research according to manufacturer's instructions. This assay assesses malondialdehyde (MDA) as an indicator of lipid oxidation. Briefly, samples were incubated with $\mathrm{N}$-methyl-2-phenylindole to yield a stable chromophore with maximal absorbance at $586 \mathrm{~nm}$ and MDA concentrations were quantified from standard curve obtained with MDA standard (1,1,3,3-Tetramethoxypropane). Since IGF-1 potentially changes macrophage-mediated LDL uptake we normalized LDL oxidation levels to LDL amounts in the conditioned media using a human ApoB ELISA kit from Cell Biolabs.

\section{OxLDL Quantification}

Mouse OxLDL levels in serum were quantified using a commercially available ELISA (Cloud-Clone Corp., Houston, TX) in accordance with manufacturer's instructions. 


\section{Eight-isoprostane Quantification}

Serum levels of 8-isoprostane were quantified using commercially available ELISA kit (Cayman Chemical) in accordance with manufacturer's instructions.

\section{Quantitative Real-Time RT-PCR}

Total RNA extraction and real-time PCR was performed as previously described [2]. Briefly, total aortic RNA was isolated using the TriPure Isolation Reagent (Roche) followed by purification with the RNeasy mini kit (Qiagen). CDNA was synthesized using the First Strand cDNA Synthesis kit (Amersham) and used for the 40-cycle 2-step PCR with sequence-specific primer pairs in the iCycler IQ Real-Time Detection System (Bio-Rad).

\section{Foam Cell Formation Assay}

To quantify foam cells, LDL-treated macrophages were fixed with $4 \%$ paraformaldehyde for $10 \mathrm{~min}$, washed with PBS and stained for $30 \mathrm{~min}$ with the neutral lipid-sensitive stain Oil Red (5 mg/ml in 60\% isopropanol). After a short wash with $60 \%$ isopropanol and PBS, macrophages were imaged with an Olympus microscope and Oil Red was extracted by $100 \%$ isopropanol (10 min on a shaker) and absorbance was read at $571 \mathrm{~nm}$ with a microplate reader.

\section{Cytokines Assay}

Levels of pro-inflammatory cytokines were quantified with Human Inflammatory Cytokines Multi-Analyte ELISArray kit (Qiagen, Valencia, CA) in accordance with manufacturer's instructions.

\section{Statistical Analysis}

All numerical data are expressed as mean \pm SEM. Two-tailed unpaired Student $t$ tests were performed to determine statistical significance. Differences were considered significant at $P<0.05$.

\section{RESULTS}

\section{IGF-1 downregulated 12/15-LOX in atherosclerotic plaque and reduced lipid oxidation in Apoe $^{-/}$mice}

We have demonstrated previously that chronic IGF-1 infusion in Apoe ${ }^{-/-}$mice reduced atherosclerotic burden [11]. Atherosclerotic plaque from IGF-1-infused mice had decreased macrophage levels and reduced plaque lipids compared to saline-infused mice (control) [11] indicating that IGF-1 suppressed macrophage-derived foam cells. 12/15-LOX is the major pro-atherogenic enzyme mediating the formation of oxidized lipids (such as oxidized low density lipoprotein, OxLDL) [3]. We quantified plaque 12/15-LOX levels in IGF-1- and saline-infused $\mathrm{Apoe}^{-/-}$mice fed with Western diet for 12 weeks. 12/15-LOX immunopositivity was strongly detected only in the plaque cap and shoulder area (Fig.1A). 12/15-LOX immunopositivity was mainly co-localized with macrophage marker indicating that macrophages are the major source of 12/15-LOX in the plaque (Suppl.Fig1). IGF-1infused mice had a significant $67 \pm 6 \%$ reduction in plaque $12 / 15$-LOX expression compared 
to control (Fig.1B). The decrease in 12/15-LOX levels induced by IGF-1 was larger than the IGF-1-promoted reduction in plaque size and in macrophage content [11], suggesting that 12/15-LOX downregulation was not secondary to these effects.

To determine whether IGF-1 reduces formation of oxidized lipids we infused human recombinant IGF-1 (or saline, control) for 4 weeks into $\mathrm{Apoe}^{-/}$mice fed with Western diet. Our experimental protocol provided a 1.4-fold increase in total (human + mouse) serum IGF-1 levels (Fig.2A). IGF-1 reduced 12/15-LOX mRNA levels in mouse atherosclerotic aortas (by $36 \pm 4 \%$, p <0.0005) (Fig.2B). IGF-1 decreased serum OxLDL levels by $18 \%$ without changing total lipoprotein levels (Fig.2C, D). IGF-1 also significantly decreased serum levels of malondialdehyde (MDA) (Fig.2E), a general marker of lipid oxidation [15]. IGF-1 reduced serum 8-isoprostane levels, a marker of oxidative stress [38], however this effect did not reach statistical significance $(\mathrm{P}=0.10)$ (Suppl.Fig.2). These data demonstrate that IGF-1 downregulates 12/15-LOX in the atherosclerotic plaque and this effect correlates with reduced lipid oxidation.

\section{IGF-1 downregulated 12/15-LOX expression in cultured macrophages via a PI3 kinase and STAT6 transcription factor-mediated mechanism}

To further study mechanisms, human THP-1 macrophages were exposed to $0-100 \mathrm{ng} / \mathrm{ml}$ IGF-1 for $6 \mathrm{~h}$ and 12/15-LOX gene expression was analyzed by RT-PCR. IGF-1 significantly reduced 12/15-LOX mRNA levels by $48 \pm 7 \%(50 \mathrm{ng} / \mathrm{ml})$ and $55 \pm 6 \%(100 \mathrm{ng} / \mathrm{ml})($ Fig.3A). IGF-1 (50 ng/ml, 16h) significantly reduced $12 / 15$-LOX protein expression by $76 \pm 6 \%(\mathrm{n}=9)$ in THP-1 macrophages as quantified by immunoblotting with 12/15-LOX antibody (Fig. 3BC). Treatment with IGF-1 also markedly downregulated 12/15-LOX in human PBMCderived macrophages (by $83 \pm 16 \%$, P $<0.05$, Fig.3DE). Since PI3 kinase- and/or ERKdependent pathways mediate IGF-1-induced intracellular signaling [10], we tested the effects of PI3 kinase (LY2940032, $50 \mathrm{uM}$ ) and ERK (PD98059, $50 \mathrm{uM}$ ) inhibitors on IGF-1-induced 12/15-LOX downregulation. We found that pre-treatment of THP-1 macrophages with PI3 kinase inhibitor $(n=4)$ but not with ERK inhibitor $(n=4)$ partially prevented the decrease in 12/15-LOX induced by IGF-1 (Fig.3FG).These data demonstrate that a PI3 kinase-mediated pathway is involved in IGF-1's effect on 12/15-LOX expression. It has been shown that IL-4 upregulates 12/15-LOX in human monocytes and in murine macrophages via an increase in expression of signal transducer and activator of transcription 6 (STAT6) [16] [17]. IL-4-induced STAT6 activation involves phosphorylation of Tyr641; however, unphosphorylated STAT6 is also able to regulate gene expression via association with p300 activation factor [18-20]. We hypothesized that IGF-1 downregulates macrophage 12/15-LOX via a STAT6-dependent mechanism. We found that IGF-1 downregulates STAT6 protein expression in THP-1 macrophages (Fig.4A). Treatment with IGF-1 for 6-18 hours did not change STAT6 gene expression levels (Suppl.Fig.3) suggesting that a posttransciptional mechanism was responsible for STAT6 downregulation by IGF-1. A brief time treatment with IL-4 (30 min) induces STAT6 phosphorylation, however macrophage treatment with IGF-1 did not induce this effect (Suppl.Fig.3B). To demonstrate that IGF-1-induced STAT6 downregulation is sufficient to reduce 12/15-LOX expression, we used STAT6-specific siRNA. A/STAT6 siRNA induced a 65\% reduction in STAT6 protein levels and this effect correlated with a significant downregulation of 12/15- 
LOX (Fig.4B). Taken together these data suggest that IGF-1 downregulates 12/15-LOX in THP-1 macrophages, at least in part via a PI3 kinase-dependent and STAT6-mediated mechanism.

\section{IGF-1 suppressed macrophage-mediated lipid oxidation and foam cell formation via its ability to downregulate 12/15-LOX}

To determine whether IGF-1-induced downregulation of 12/15-LOX led to suppression of macrophage-mediated lipid oxidation, IGF-1-treated THP-1 macrophages or control cells were incubated with LDL ( $150 \mathrm{ug} / \mathrm{ml}$ for $48 \mathrm{~h}$ ) and lipid oxidation levels were assessed by measuring TBARS in conditioned media with data normalization to LDL amounts in the media. We found that THP-1 macrophages time-dependently increased LDL oxidation with a peak at $40-48 \mathrm{~h}$ and maximal lipid internalization at this time point occurred with 150 $\mathrm{ug} / \mathrm{ml}$ LDL (data not shown). We found that IGF-1-treated THP-1 macrophages had reduced ability to oxidize lipids: LDL incubated with IGF-1-treated cells had a $65 \pm 4 \%$ decrease in oxidation levels compared to LDL incubated with untreated macrophages (Fig.5A). Similarly, IGF-1 markedly suppressed LDL oxidation ( $79 \pm 14 \%$ decrease, $\mathrm{P}<0.05)$ induced by human PBMC-derived macrophages (Fig.5B).

Since LDL oxidation increases LDL uptake by macrophages (i.e. formation of foam cells) [21], we measured foam cell formation in IGF-1-treated or control THP-1 macrophages after incubation with LDL (Fig.5CD). IGF-1 reduced macrophage lipid internalization by $64 \pm 4 \%$ $(\mathrm{P}<0.001)$. These data demonstrate that IGF-1-induced 12/15-LOX downregulation was associated with a reduction in macrophage-mediated lipid oxidation and suppressed foam cell formation.

We have reported previously that IGF-1-induced atheroprotective effect was associated with reduced inflammatory response [11]. To assess IGF-1's effect on macrophage inflammatory profile we quantified levels of 12 inflammatory cytokines in conditioned media of IGF-1treated (or control) THP-1 macrophages (Table). IGF-1 significantly decreased expression of IL-1 $\beta$, IFN $\gamma$ and granulocyte macrophage colony-stimulating factor (GM-CSF). These results suggest that suppression of macrophage-induced cytokines as well as 12/15-LOX downregulation contribute to reduced atherosclerotic burden in IGF-1-infused Apoe-null mice.

We isolated peritoneal macrophages from 12/15-LOX-deficient and control (WT) mice and used these cells to test IGF-1's effect on cell-mediated lipid oxidation and foam cell formation. 12/15-LOX deficiency induced a dramatic reduction in cell-mediated LDL oxidation and foam cell formation (Fig.6). IGF-1 reduced LDL oxidation and formation of foam cells by peritoneal macrophages isolated from WT mice but these effects were blocked in 12/15-LOX-deficient macrophages (Fig.6). These data demonstrate that 12/15-LOX plays the key role in LDL oxidation and formation of macrophage-derived foam cells and that the ability of IGF-1 to reduce LDL oxidation, lipid uptake and foam cell formation is dependent on its ability to downregulate $12 / 15$-LOX. 


\section{DISCUSSION}

IGF-1 is a growth factor that exerts multiple beneficial effects on vascular cells and in animal models, including an anti-oxidant effect, prevention of cell apoptosis and an antiinflammatory effect [22]. IGF-1 is approved for the treatment of growth hormone deficiency in children [23], and IGF-1 is in development for the treatment of muscle disorders [24] and neurodegenerative diseases [25]. Basic research data and clinical reports suggest that IGF-1 also has potential as a novel anti-atherosclerotic drug with the ability to reduce atherosclerotic plaque size and increase plaque stability. Here we reported that IGF-1 suppressed 12/15-LOX expression in the atherosclerotic plaque and reduced lipid oxidation in Apoe $^{-/}$mice. To identify mechanisms mediating these IGF-1 effects and also to obtain physiologically relevant data we focused on using human THP-1 macrophages and human PBMC-derived macrophages. We found that IGF-1 reduces 12/15-LOX in THP-1 cells at least in part via a PI3 kinase- and STAT6 transcription factor-mediated signaling mechanism and that IGF-1 suppressed the ability of macrophages to oxidize lipids and to form foam cells. IGF-1-induced effects on lipid oxidation and foam cell formation were blocked in $12 / 15-\mathrm{LOX}^{-/-}$murine macrophages suggesting that they are 12/15-LOX-dependent. We have demonstrated that IGF-1 downregulates 12/15-LOX and suppressed LDL oxidation also in human PBMC-derived macrophages consistent with our major findings obtained with THP-1 cells. Macrophages are the primary source of 12/15-LOX in mouse tissues [7] however smooth muscle and other vascular cells are known to express 12/15-LOX [3]. IGF-1 may regulate 12/15-LOX expression in non-myeloid cells and this effect could also contribute to IGF-1-induced suppression of atherosclerosis.

Uptake of oxidized lipids by macrophages in the early stages of atherosclerosis may be considered to be a protective mechanism providing the removal of cytotoxic lipoproteins, however, the progressive uptake of OxLDL leads to the formation of foam cells and thus, to the growth of atherosclerotic lesions. Internalized lipoproteins were delivered to lysosomes where the cholesteryl ester is hydrolyzed to free cholesterol and free cholesterol can be released from the cell. The difference in the amount of cholesterol accumulated within the cellular compartments and the cholesterol amount released is believed to induce foam cell formation [26]. There is a growing body of evidence that suggests that lipoxygenases including 12/15-LOX play an essential role in LDL oxidation and in formation of macrophage-derived foam cells [27]. Our current finding that peritoneal macrophages isolated from 12/15-LOX-null mice have reduced ability to oxidize lipids and to form foam cells is consistent with a key role of 12/15-LOX in mediating these effects. 12/15-LOXdependent LDL oxidation involves lipoxygenase translocation to the plasma membrane, which activates the oxygenase activity of enzyme [28] [29]. Interestingly, we noticed that brief sonication of macrophage lysates dramatically increased immunodetection of 12/15LOX with Westerns (data not shown) suggesting membrane-associated 12/15-LOX localization in THP-1 cells.

We have demonstrated previously that IGF-1 infusion into Apoe ${ }^{-/}$mice did not change total cholesterol levels and also IGF-1 did not affect individual lipoprotein fractions (VLDL, IDL/LDL, and HDL) [11]. In the current study we measured serum levels of ApoB (the principal LDL lipoprotein) and we found that IGF-1 has no effect on total lipoprotein levels. 
However, it was evident that IGF-1 reduced OxLDL levels suggesting that formation of this pro-atherogenic oxidized lipoprotein (which mainly depends on 12/15-LOX activity) was specifically suppressed by IGF-1.

We have shown previously that OxLDL was co-localized with apoptotic cells in human advanced atherosclerotic plaque [9] and that OxLDL induces cell apoptosis and oxidative stress in vitro [30] [31]. We also reported that IGF-1 reduced apoptosis and suppressed oxidative stress in atherosclerotic plaque [12] [11]. Our current findings showing that IGF-1 reduces OxLDL, 8-isoprostane and MDA levels in $\mathrm{Apoe}^{-/-}$mice, in view of the fact that suppression of 12/15-LOX in vivo reduces cell apoptosis [32] and oxidative stress [33] [34], suggest that 12/15-LOX downregulation directly (or via reduction in oxidized lipids levels) mediates IGF-1-dependent anti-oxidant and anti-apoptotic effects.

Therapies directed towards suppression of 12/15-LOX activity are a novel developing approach to control cardiovascular disease (CVD) [35], treat asthma and rheumatoid arthritis [36] and combat stroke and other neurodegenerative diseases [37]. Progress in development of anti-atherosclerotic drugs targeting 12/15-LOX is partially limited by the lack of basic research data describing cell-specific molecular mechanisms of 12/15-LOX regulation. Our finding that IGF-1 downregulates STAT6 expression and that siRNA-mediated downregulation of STAT-6 is sufficient to decrease 12/15-LOX expression suggest strongly that IGF-1-induced decrease in STAT6 mediates its ability to decrease 12/15-LOX. IGF-1 did not alter Tyr641 phosphorylation of STAT6. Several molecules known to activate/ suppress STAT6 (such as SOCS molecules, p100 STAT6 co-activator or p300 activation factor) could potentially mediate IGF-1's effect on STAT6 and on 12/15-LOX expression. Identification of such mechanisms regulating 12/15-LOX expression in macrophages could offer novel targets for therapeutic interventions in CVD and other types of inflammatory diseases.

In summary, we have shown that IGF-1 decreased 12/15-LOX plaque immunopositivity and serum OxLDL levels in Apoe ${ }^{-/}$mice. IGF-1 reduced 12/15-LOX protein and mRNA levels in cultured THP-1 macrophages and IGF-1 also decreased expression of STAT6 transcription factor. We have shown that IGF-1 reduces macrophage 12/15-LOX in part via a PI3 kinase- and STAT6-dependent transcriptional mechanism. IGF-1 suppressed THP-1 macrophage ability to oxidize lipids and form foam cells. IGF-1 similarly reduced LDL oxidation and formation of foam cells by wild type murine peritoneal macrophages, however these effects were blocked in 12/15-LOX-null macrophages suggesting that the ability of IGF-1 to reduce LDL oxidation and foam cells depends on its ability to downregulate 12/15LOX. Overall our data demonstrate that IGF-1 reduces lipid oxidation and foam cell formation via downregulation of $12 / 15-\mathrm{LOX}$ and this mechanism may play a major role in the anti-atherosclerotic effects of IGF-1.

\section{Supplementary Material}

Refer to Web version on PubMed Central for supplementary material. 


\section{ACKNOWLEDGMENTS}

This work was supported by grants from the National Institute of Health 1-R21-HL113705 (S.S.), 5-R01HL070241 (P.D.), 2-RO1-HL080682 (P.D.), 1-U54 GM104940 (P.D.) and American Heart Association Grant-inAid 13GRNT17230069 (S.S.) and grant from Tulane University Pilot Research Program (S.S.).

\section{Abbreviations}

LDL low density lipoprotein

OxLDL $\quad$ oxidized low density lipoprotein

IGF-1 insulin-like growth factor I

12/15-LOX 12/15-lipoxygenase

MDA malondialdehyde

\section{REFERENCES}

1. Parthasarathy S, Steinberg D, Witztum JL. The role of oxidized low-density lipoproteins in the pathogenesis of atherosclerosis. Annu Rev Med. 1992; 43:219-225. [PubMed: 1580586]

2. Yu XH, et al. Foam cells in atherosclerosis. Clin Chim Acta. 2013; 424:245-252. [PubMed: 23782937]

3. Funk CD. Lipoxygenase pathways as mediators of early inflammatory events in atherosclerosis. Arterioscler Thromb Vasc Biol. 2006; 26(6):1204-1206. [PubMed: 16709954]

4. Cyrus T, et al. Disruption of the 12/15-lipoxygenase gene diminishes atherosclerosis in apo Edeficient mice. J Clin Invest. 1999; 103(11):1597-1604. [PubMed: 10359569]

5. Cyrus T, et al. Absence of 12/15-lipoxygenase expression decreases lipid peroxidation and atherogenesis in apolipoprotein e-deficient mice. Circulation. 2001; 103(18):2277-2282. [PubMed: 11342477]

6. George J, et al. 12/15-Lipoxygenase gene disruption attenuates atherogenesis in LDL receptordeficient mice. Circulation. 2001; 104(14):1646-1650. [PubMed: 11581143]

7. Huo Y, et al. Critical role of macrophage 12/15-lipoxygenase for atherosclerosis in apolipoprotein E-deficient mice. Circulation. 2004; 110(14):2024-2031. [PubMed: 15451785]

8. Okura Y, et al. Oxidized low-density lipoprotein is associated with apoptosis of vascular smooth muscle cells in human atherosclerotic plaques. Circulation. 2000; 102(22):2680-2686. [PubMed: 11094032]

9. Okura Y, et al. Decreased expression of insulin-like growth factor-1 and apoptosis of vascular smooth muscle cells in human atherosclerotic plaque. J Mol Cell Cardiol. 2001; 33(10):1777-1789. [PubMed: 11603921]

10. Delafontaine P, Song YH, Li Y. Expression, regulation, and function of IGF-1, IGF-1R, and IGF-1 binding proteins in blood vessels. Arterioscler Thromb Vasc Biol. 2004; 24(3):435-444. [PubMed: 14604834]

11. Sukhanov S, et al. IGF-1 reduces inflammatory responses, suppresses oxidative stress, and decreases atherosclerosis progression in ApoE-deficient mice. Arterioscler Thromb Vasc Biol. 2007; 27(12):2684-2690. [PubMed: 17916769]

12. Sukhanov S, et al. Differential requirement for nitric oxide in IGF-1-induced anti-apoptotic, antioxidant and anti-atherosclerotic effects. FEBS Lett. 2011; 585(19):3065-3072. [PubMed: 21872589]

13. Shai SY, et al. Smooth muscle cell-specific insulin-like growth factor-1 overexpression in Apoe-/mice does not alter atherosclerotic plaque burden but increases features of plaque stability. Arterioscler Thromb Vasc Biol. 2010; 30(10):1916-1924. [PubMed: 20671230] 
14. Titterington JS, et al. Growth hormone-releasing peptide-2 suppresses vascular oxidative stress in ApoE-/- mice but does not reduce atherosclerosis. Endocrinology. 2009; 150(12):5478-5487. [PubMed: 19819949]

15. Del Rio D, Stewart AJ, Pellegrini N. A review of recent studies on malondialdehyde as toxic molecule and biological marker of oxidative stress. Nutr Metab Cardiovasc Dis. 2005; 15(4):316328. [PubMed: 16054557]

16. Conrad DJ, Lu M. Regulation of human 12/15-lipoxygenase by Stat6-dependent transcription. Am J Respir Cell Mol Biol. 2000; 22(2):226-234. [PubMed: 10657944]

17. Heydeck D, et al. Interleukin-4 and-13 induce upregulation of the murine macrophage 12/15lipoxygenase activity: evidence for the involvement of transcription factor STAT6. Blood. 1998; 92(7):2503-2510. [PubMed: 9746791]

18. Cui X, et al. Unphosphorylated STAT6 contributes to constitutive cyclooxygenase-2 expression in human non-small cell lung cancer. Oncogene. 2007; 26(29):4253-4260. [PubMed: 17237818]

19. Yan SJ, et al. Unphosphorylated STAT and heterochromatin protect genome stability. FASEB journal : official publication of the Federation of American Societies for Experimental Biology. 2011; 25(1):232-241. [PubMed: 20847228]

20. Yang J, Stark GR. Roles of unphosphorylated STATs in signaling. Cell research. 2008; 18(4):443451. [PubMed: 18364677]

21. Lougheed M, Steinbrecher UP. Mechanism of uptake of copper-oxidized low density lipoprotein in macrophages is dependent on its extent of oxidation. J Biol Chem. 1996; 271(20):11798-11805. [PubMed: 8662601]

22. Higashi Y, et al. Aging, atherosclerosis, and IGF-1. J Gerontol A Biol Sci Med Sci. 2012; 67(6): 626-639. [PubMed: 22491965]

23. Chernausek SD, et al. Long-term treatment with recombinant insulin-like growth factor (IGF)-I in children with severe IGF-I deficiency due to growth hormone insensitivity. J Clin Endocrinol Metab. 2007; 92(3):902-910. [PubMed: 17192294]

24. Clemmons DR. Role of IGF-I in skeletal muscle mass maintenance. Trends Endocrinol Metab. 2009; 20(7):349-356. [PubMed: 19729319]

25. Gasperi M, Castellano AE. Growth hormone/insulin-like growth factor I axis in neurodegenerative diseases. J Endocrinol Invest. 2010; 33(8):587-591. [PubMed: 20930497]

26. Jones NL, Reagan JW, Willingham MC. The pathogenesis of foam cell formation: modified LDL stimulates uptake of co-incubated LDL via macropinocytosis. Arterioscler Thromb Vasc Biol. 2000; 20(3):773-781. [PubMed: 10712403]

27. Takahashi Y, Zhu H, Yoshimoto T. Essential roles of lipoxygenases in LDL oxidation and development of atherosclerosis. Antioxid Redox Signal. 2005; 7(3-4):425-431. [PubMed: 15706089]

28. Brinckmann R, et al. Membrane translocation of 15-lipoxygenase in hematopoietic cells is calcium-dependent and activates the oxygenase activity of the enzyme. Blood. 1998; 91(1):64-74. [PubMed: 9414270]

29. Zhu H, et al. Low density lipoprotein receptor-related protein-mediated membrane translocation of 12/15-lipoxygenase is required for oxidation of low density lipoprotein by macrophages. $\mathrm{J}$ Biol Chem. 2003; 278(15):13350-13355. [PubMed: 12566436]

30. Li Y, et al. Insulin-like growth factor-1 receptor activation inhibits oxidized LDL-induced cytochrome $\mathrm{C}$ release and apoptosis via the phosphatidylinositol 3 kinase/Akt signaling pathway. Arterioscler Thromb Vasc Biol. 2003; 23(12):2178-2184. [PubMed: 14551153]

31. Higashi Y, et al. A redox-sensitive pathway mediates oxidized LDL-induced downregulation of insulin-like growth factor-1 receptor. J Lipid Res. 2005; 46(6):1266-1277. [PubMed: 15805544]

32. Song L, et al. Inhibition of 12/15 lipoxygenase by baicalein reduces myocardial ischemia/ reperfusion injury via modulation of multiple signaling pathways. Apoptosis. 2014; 19(4):567580. [PubMed: 24248985]

33. Zhao L, et al. 12/15-Lipoxygenase gene disruption and vitamin $\mathrm{E}$ administration diminish atherosclerosis and oxidative stress in apolipoprotein $\mathrm{E}$ deficient mice through a final common pathway. Prostaglandins Other Lipid Mediat. 2005; 78(1-4):185-193. [PubMed: 16303615] 
34. Chinnici CM, et al. Absence of 12/15 lipoxygenase reduces brain oxidative stress in apolipoprotein E-deficient mice. Am J Pathol. 2005; 167(5):1371-1377. [PubMed: 16251421]

35. Siu D. A new way of targeting to treat coronary artery disease. J Cardiovasc Med (Hagerstown). 2010; 11(1):1-6. [PubMed: 19829140]

36. Chen S. Natural products triggering biological targets--a review of the antiinflammatory phytochemicals targeting the arachidonic acid pathway in allergy asthma and rheumatoid arthritis. Curr Drug Targets. 2011; 12(3):288-301. [PubMed: 20955151]

37. van Leyen K, et al. Novel lipoxygenase inhibitors as neuroprotective reagents. J Neurosci Res. 2008; 86(4):904-909. [PubMed: 17960827]

38. Patrignani P, Tacconelli S. Isoprostanes and other markers of peroxidation in atherosclerosis. Biomarkers. 2005; 10(1):S24-S29. [PubMed: 16298908] 


\section{Highlights}

- Insulin-like growth factor I (IGF-1) suppressed 12/15-lipoxygenase, reduced lipid oxidation, foam cell formation and prevented atherosclerosis in vivo

- IGF-1 suppressed 12/15-lipoxygenase in THP-1 macrophages and also IGF-1 decreased ability of these cells to oxidize lipids and form foam cells

- Both STAT6 transcription factor and PI3 kinase were involved in IGF-1induced 12/15-lipoxygenase downregulation in THP-1 macrophages

- 12/15-lipoxygenase downregulation mediates IGF-1' effect on lipid oxidation and foam cells 

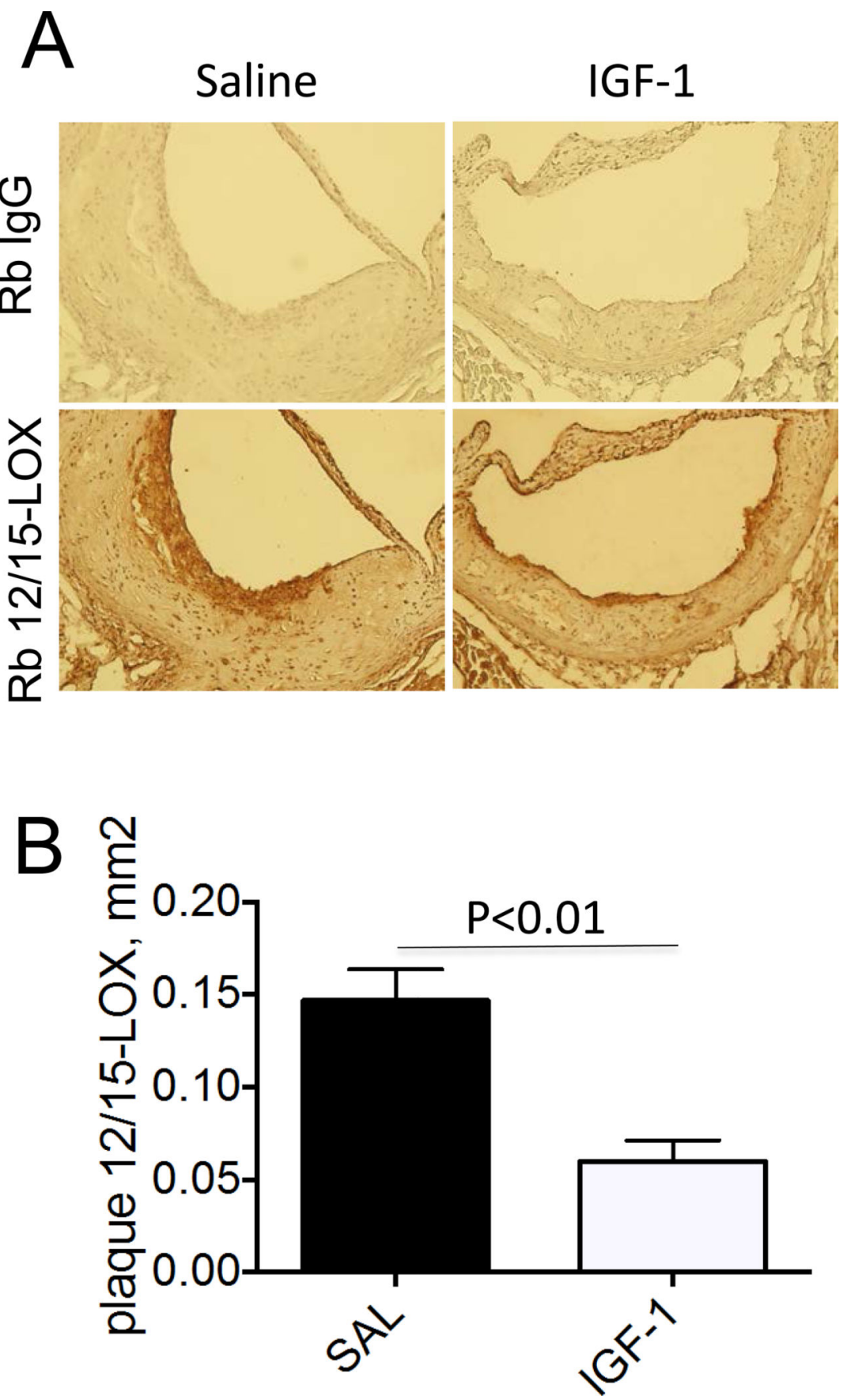

Figure 1. IGF-1 downregulated 12/15-LOX in atherosclerotic plaque

Apoe-/- mice were infused with human IGF-1 or with saline (control) and fed with Western type diet for 12 weeks. Serial cross-sections were obtained through aortic valves, stained with rabbit 12/15-LOX antibody (or with "normal" rabbit IgG, negative control), developed with a/rabbit-biotin/streptavidin-horseradish peroxidase system and counterstained with hematoxylin. A, Representative images. B, Quantative data. 
A B
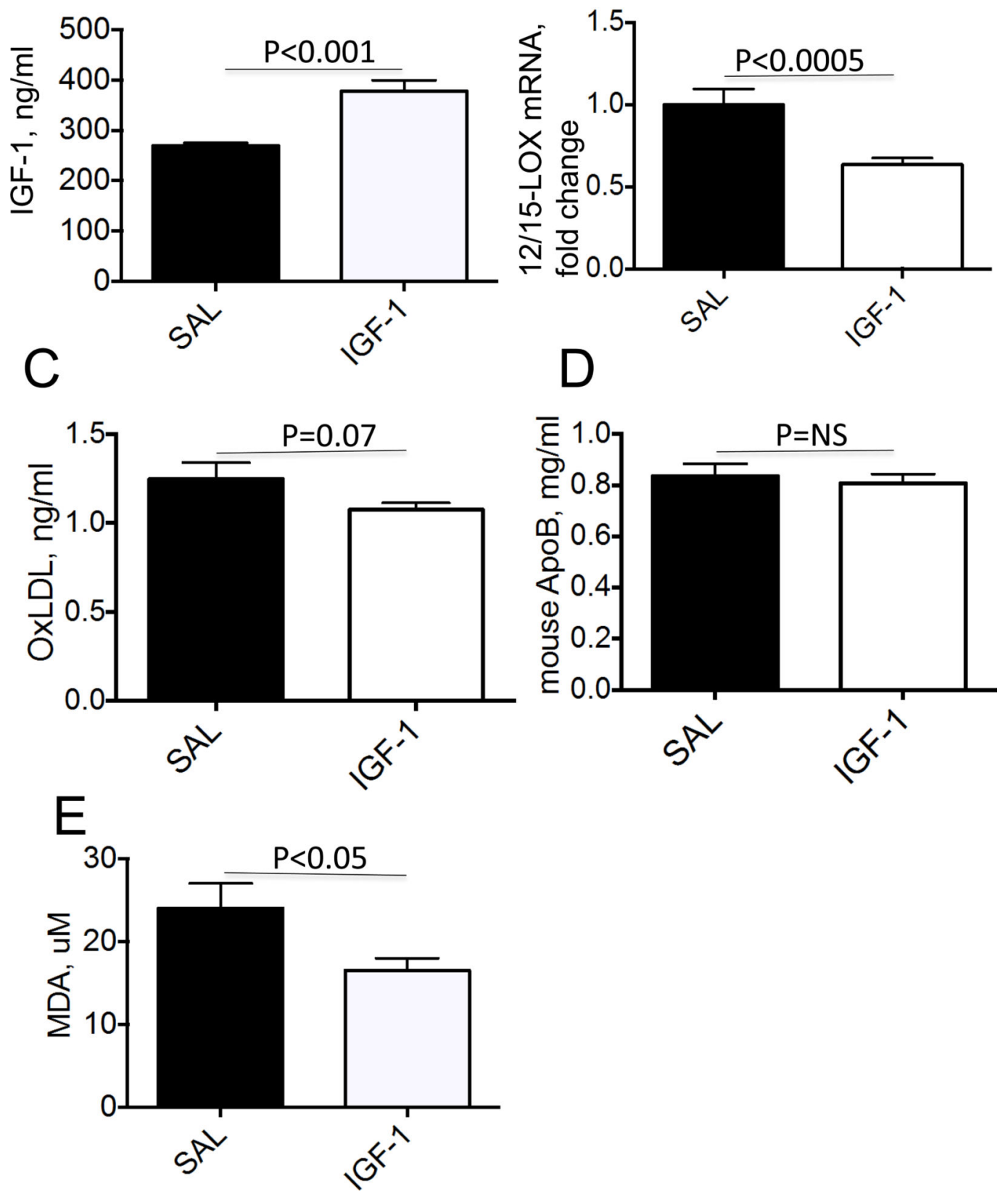

Figure 2. IGF-1 downregulated 12/15-LOX and reduced lipid oxidation in Apoe $^{-/-}$mice Mice were fed with Western type diet and infused with human IGF-1 or with saline (control). A, Total (mouse+human) serum IGF-1 levels. B, 12/15-LOX mRNA levels in atherosclerotic aortas. C, Serum OxLDL levels. D, Serum ApoB levels. E, Serum levels of malondialdehyde (MDA), an index of lipid oxidation. 
A
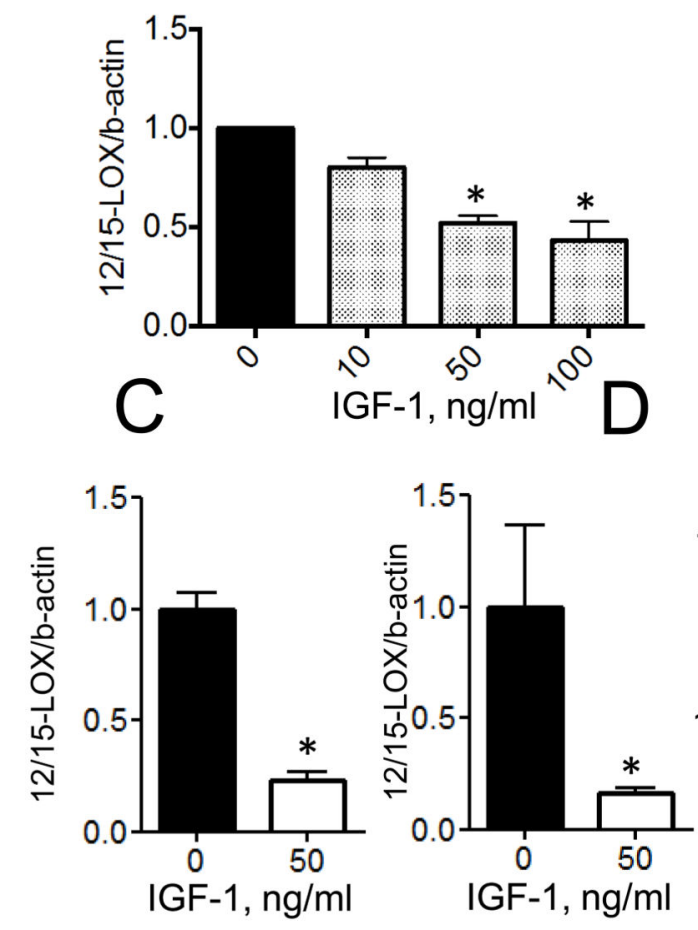
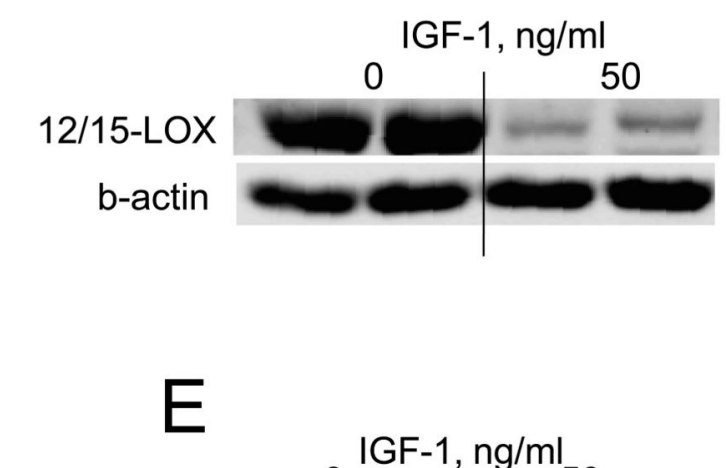

F
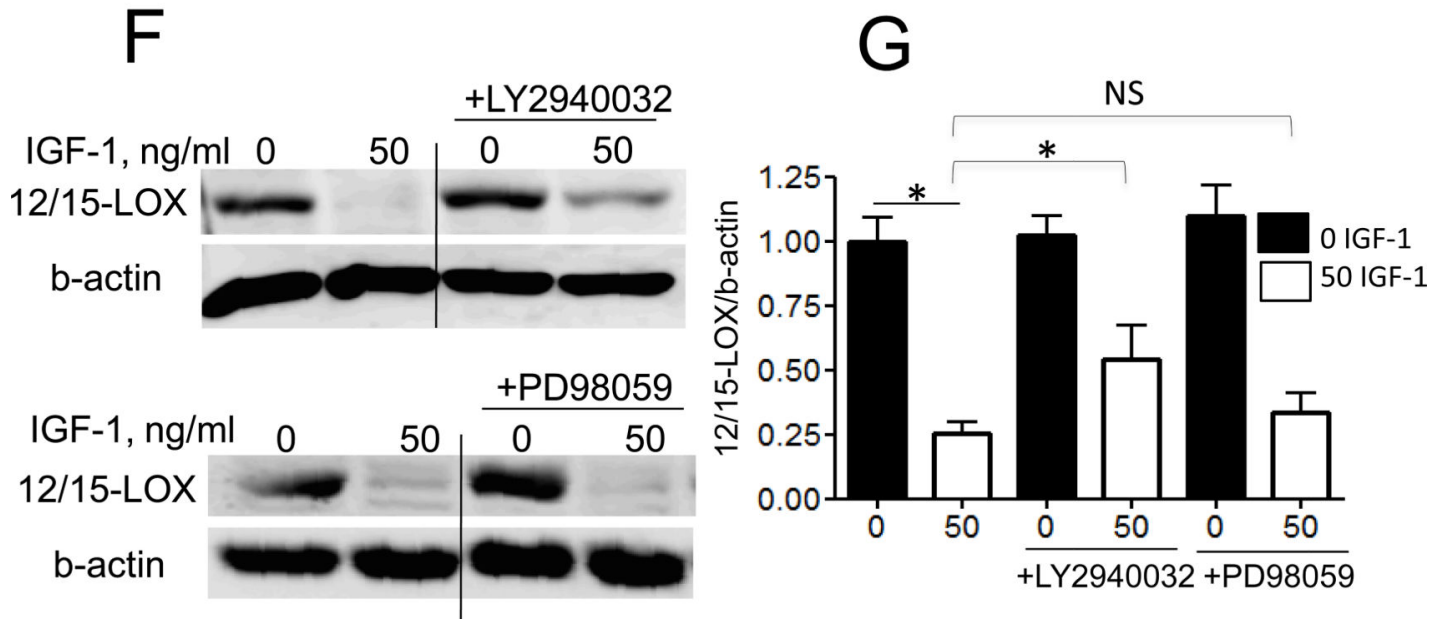

Figure 3. IGF-1 decreased 12/15-LOX expression in THP-1 macrophages and in human PBMCderived macrophages. IGF-1 decreased 12/15-LOX in THP-1 cells via PI3 kinase-dependent mechanism

THP-1 macrophages were treated with IGF-1 and 12/15-LOX mRNA levels were measured with real time-RT-PCR (A) and protein expression with immunoblotting (B, C). IGF-1 downregulates 12/15-LOX in human PBMC-derived macrophages (D, E). THP-1 pretreatment with PI3 kinase inhibitor (LY2940032) partially prevented 12/15-LOX downregulation, however pre-treatment with ERK inhibitor (PD98059) had no effect (F,G). 
A
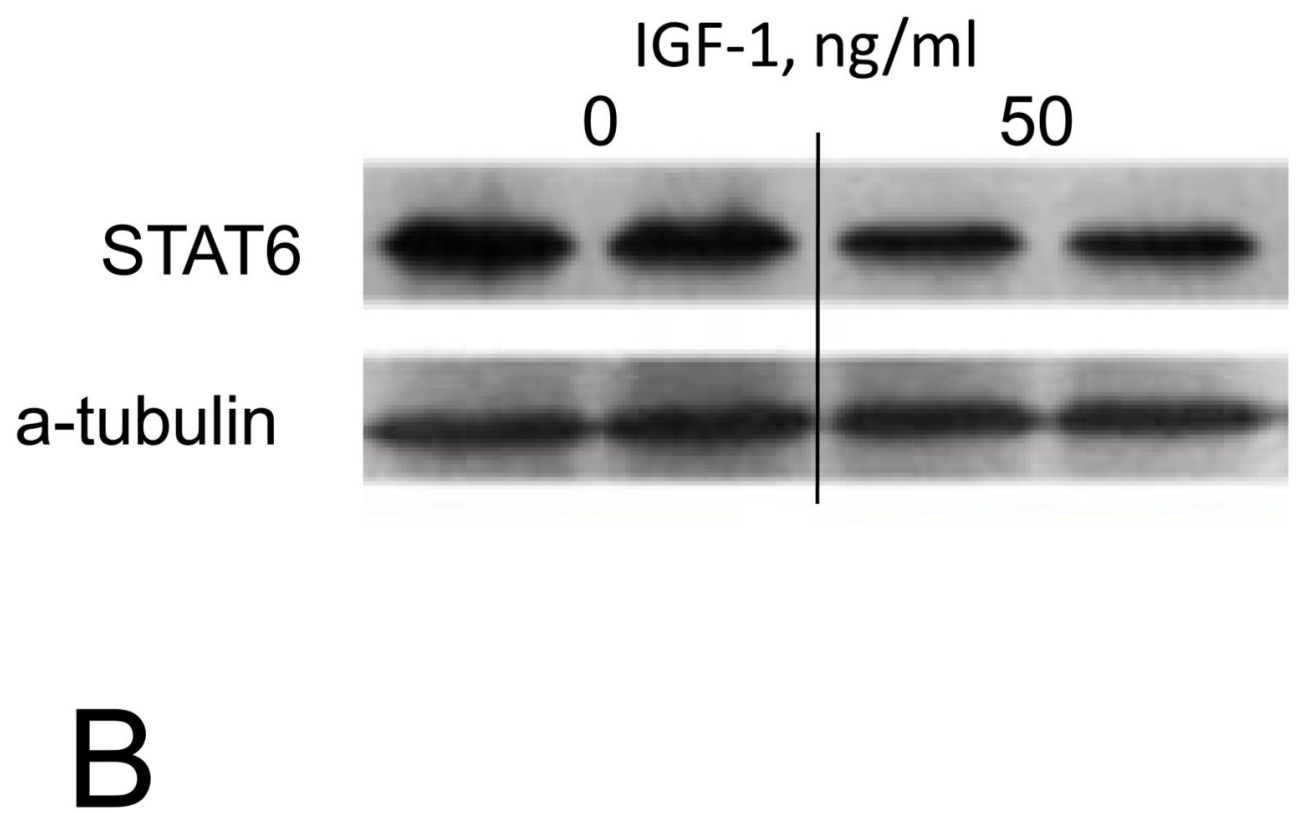

\section{Scr siRNA}

\section{a/STAT6 siRNA}

\section{STAT6}

\section{2/15-LOX}

\section{b-actin}
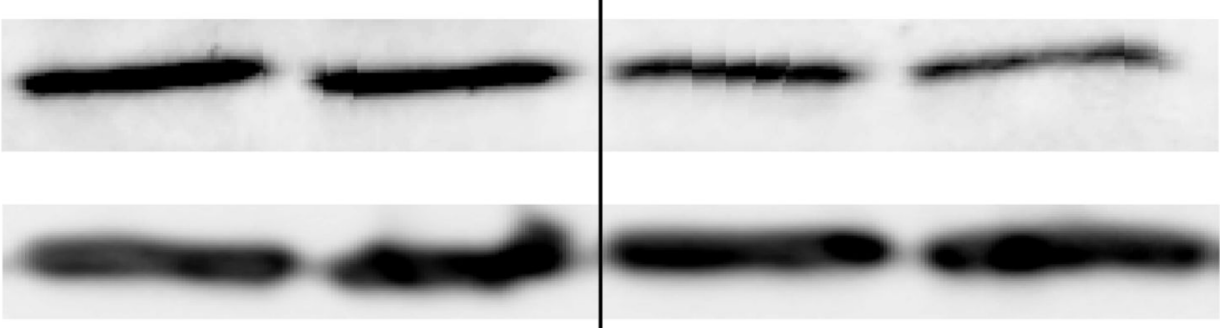

Figure 4. STAT6 transcription factor is involved in IGF-1-induced 12/15-LOX downregulation A, THP-1 macrophages were treated with IGF-1 and STAT6 protein expression was measured with immunoblotting. B, THP-1 macrophages were transfected with STAT6targeted siRNA (or with scrambled siRNA, scr siRNA, negative control) and STAT6 and 12/15-LOX protein expression were measured with immunoblotting. 
A

C
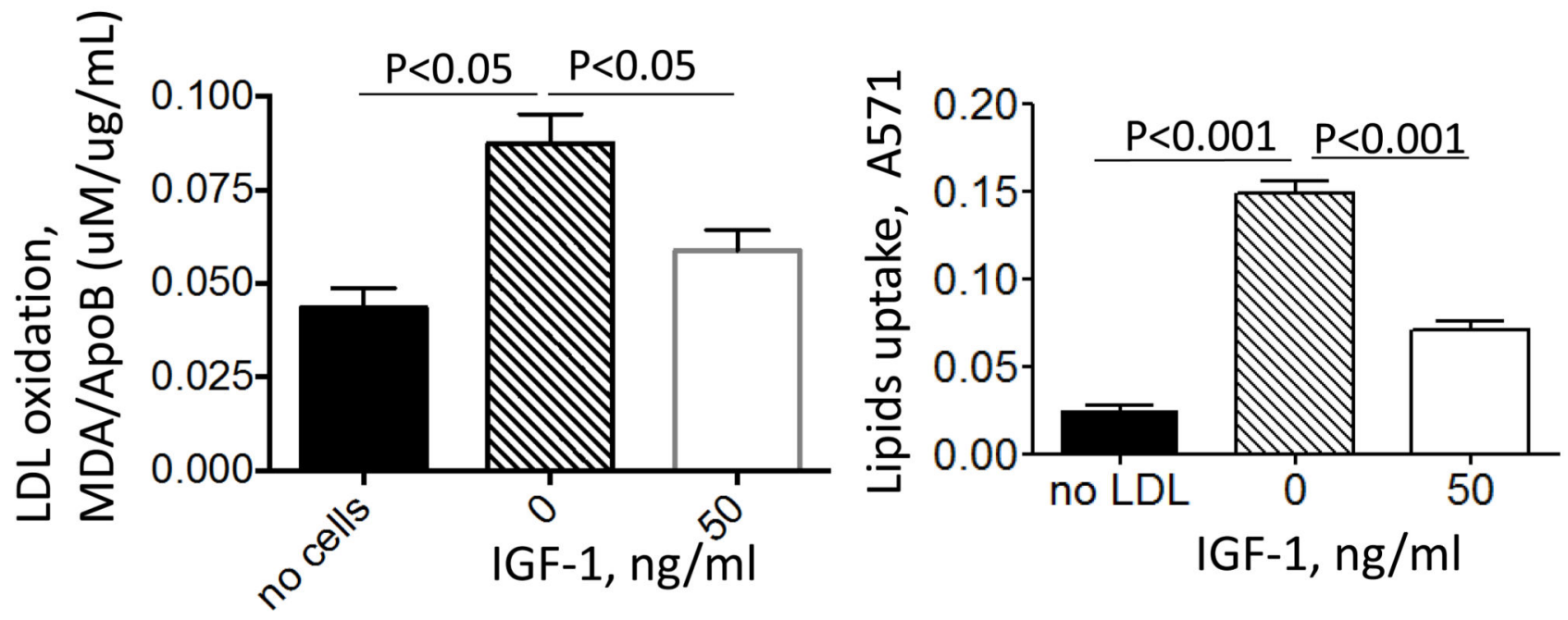

B

D
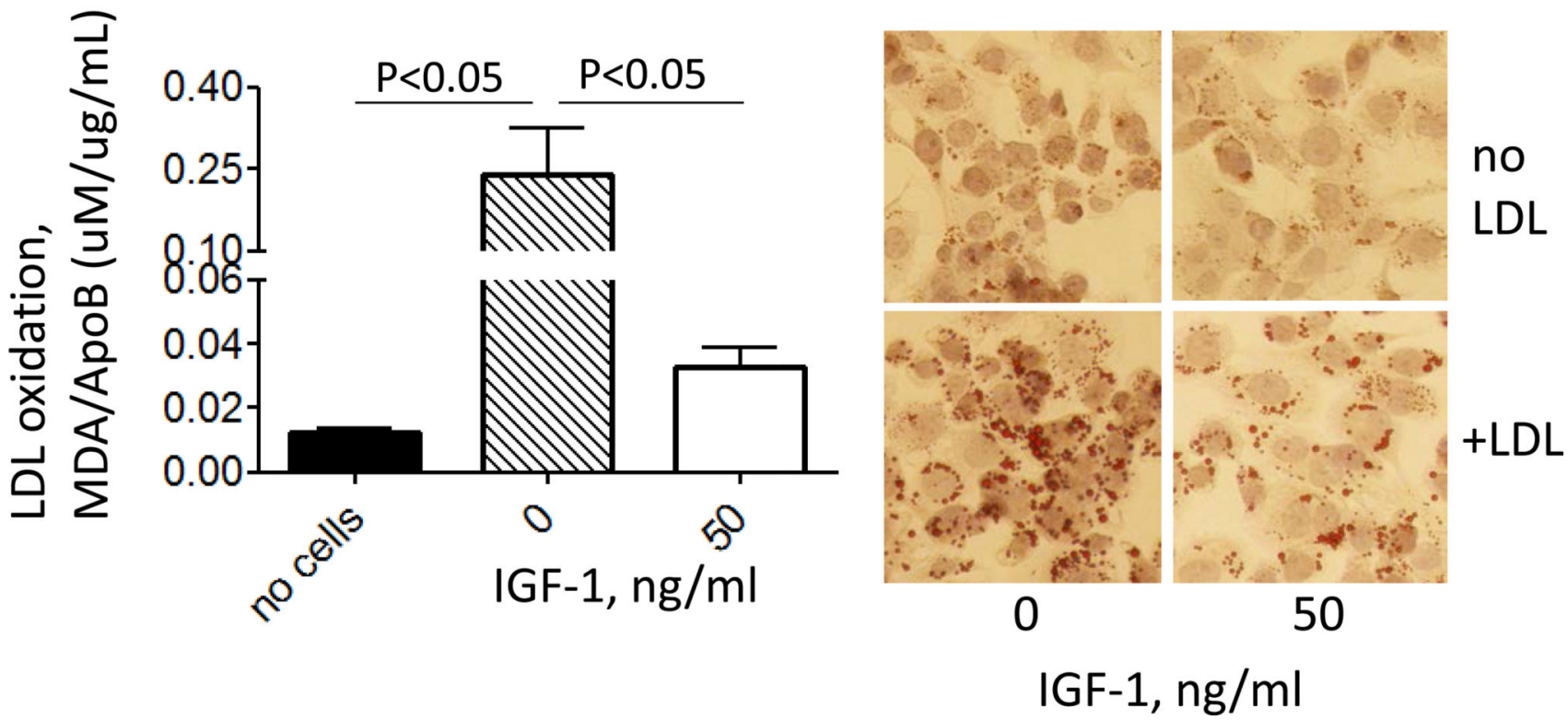

Figure 5. IGF-1 decreased lipid oxidation in THP-1 macrophages and in PBMC-derived macrophages. IGF-1 suppressed lipid uptake in THP-1 macrophages

THP-1 macrophages (A) or PBMC-derived macrophages (B) were treated with IGF-1 followed by incubation with LDL and lipid oxidation was assessed by TBARS assay. C, LDL-treated THP-1 macrophages were stained with Oil Red, imaged with a microscope and lipid uptake was quantified after extraction of Oil Red and measurement of absorbance at $571 \mathrm{~nm}$. D, representative images. 
A

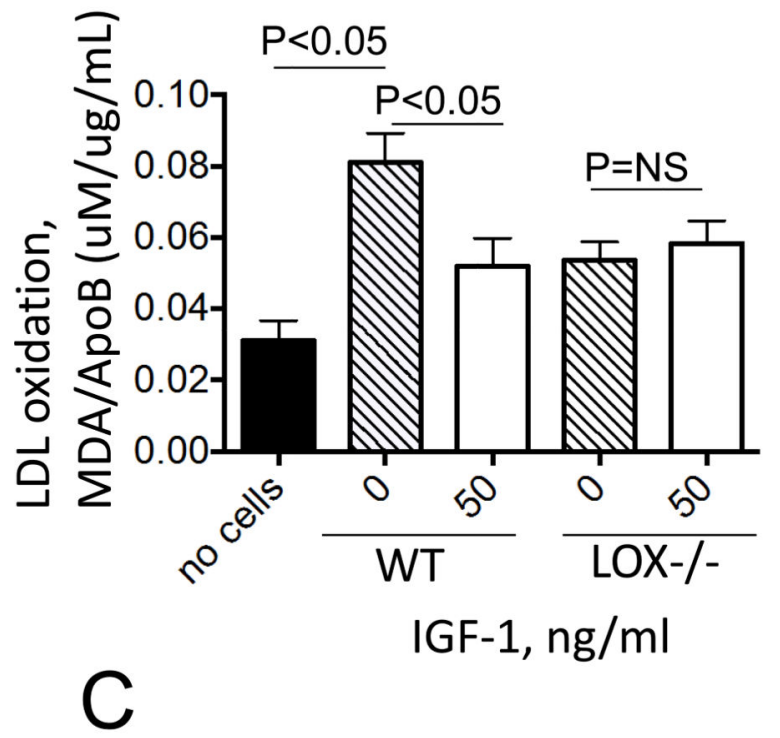

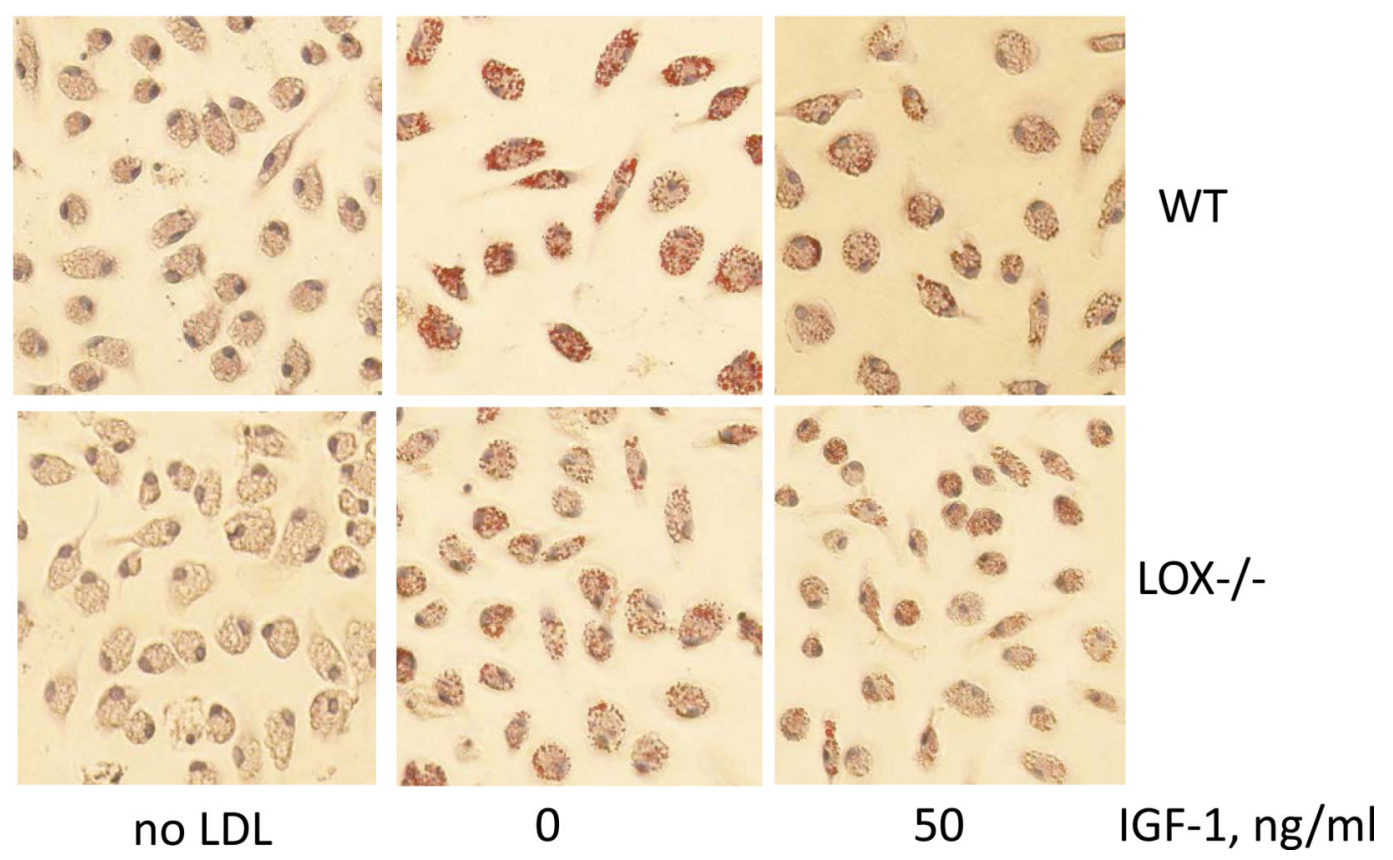

Figure 6. IGF-1 suppressed lipid oxidation and foam cell formation via its ability to downregulate 12/15-LOX

Peritoneal macrophages were isolated from 12/15 LOX-null mice or from wild-type mice (WT) and treated with IGF-1 followed by incubation with LDL. A, Lipid oxidation (TBARS assay), B, Lipid uptake (Oil Red assay), C, representative images. 


\section{Table}

IGF-1 effect on pro-inflammatory cytokine expression by THP-1 macrophages (ng per ml of conditioned media)

\begin{tabular}{|l|l|l|}
\hline Cytokines & Control & IGF-1 \\
\hline IL1a & $0.42 \pm 0.01$ & $0.44 \pm 0.01$ \\
IL1 $\beta$ & $1.01 \pm 0.02$ & $0.83 \pm 0.01 *$ \\
IL2 & $0.67 \pm 0.07$ & $0.81 \pm 0.08$ \\
IL4 & $0.41 \pm 0.01$ & $0.40 \pm 0.01$ \\
IL6 & $0.415 \pm 0.01$ & $0.418 \pm 0.004$ \\
IL8 & $26.47 \pm 2.21$ & $21.36 \pm 1.21$ \\
IL10 & $0.42 \pm 0.01$ & $0.41 \pm 0.01$ \\
IL12 & $0.39 \pm 0.01$ & $0.395 \pm 0.006$ \\
IL17A & $0.497 \pm 0.021$ & $0.461 \pm 0.014$ \\
IFN- $\gamma$ & $0.502 \pm 0.013$ & $0.445 \pm 0.013^{*}$ \\
TNF- $\alpha$ & $0.69 \pm 0.02$ & $0.52 \pm 0.02$ \\
GM-CSF & $0.80 \pm 0.05$ & $0.72 \pm 0.02^{*}$ \\
\hline
\end{tabular}

P $<0.05$ vs. Control 